\title{
Recyclable Porphyrin Catalyst with Core-shell Nanostructure
}

\author{
Bo Gyu Choi, Soo Y. Ko, Wonwoo Nam, , and Byeongmoon Jeong* \\ Department of Chemistry, Division of Nano Sciences, Ewha Womans University, Seoul 120-750, Korea \\ *E-mail:wwnam@ewha.ac.kr,bjeong@ewha.ac.kr \\ Received May 12, 2005
}

\begin{abstract}
In the search for a simple preparation method of heterogeneous catalyst, the iron porphyrins were coordinated bonded to the surface of a polymeric core-shell nanosphere. The heterogeneous catalyst was characterized by FT-IR, scanning electron microscope, and UV-vis spectrophotometer. The iron porphyrin bound core-shell nanospheres was about $470 \mathrm{~nm}$ in diameter and their catalytic activity for cyclohexene oxidation was similar to a homogeneous iron porphyrin in a solvent composition range of $25-75 \%$ acetonitrile/water (v/v). In addition, they could be recovered by simple centrifugation and their catalytic activity was maintained more than the third cycle.
\end{abstract}

Key Words : Iron porphyrin, Nanocatalyst, Recyclability, Core-shell, Nanosphere

\section{Introduction}

Polymers have been used as a heterogeneous catalytic support since the pioneering work by Merrifield's solid phase peptide synthesis. ${ }^{1}$ The polymer bound catalyst provide several advantages over a homogeneous catalyst such as easy separation from the reaction mixture and recyclability of the catalyst. They are critical points for the fine chemicals or pharmaceuticals industry where a trace amount of the residual catalyst may induce serious toxicity and the cost of the catalyst is highly expensive. ${ }^{2}$ Traditionally, polystyrene beads have been used as the polymer support. The chloromethyl styrene was copolymerized with styrene to prepare the bead. The nucleophilic reaction between the catalyst and the chloromethyl group gives the catalyst-bound polystyrene bead. However, tedious chemical reactions for the preparation of the polymer-supported catalyst and the leaching out of the catalyst during the recycling have been a concern.

In order for the catalyst to contact effectively with the reagents, several requirements must be satisfied. First, it should be well suspended in the reaction medium during the reaction. Therefore, the surface properties of heterogeneous catalyst should be compatible with the reaction medium. Second, the catalyst should be localized to the surface to effectively react with the reagent.

Based on these considerations, we designed a nanosphere with core-shell structure. The core consisting of poly(styrene-co-N-isopropylacrylamide) gives the structural rigidity in the reaction medium. Whereas, the shell consisting of poly( $\mathrm{N}$-isopropylacrylamide-co-vinyl pyridine) has the ligands (pyridine) to bind to the catalyst. Metalloporphyrins have been extensively used as catalysts for chemical and biochemical reactions. ${ }^{3,4}$ However, they are expensive and hard to synthesize. Therefore, the preparation of a recyclable metalloporphyrin bound nanosphere is an important issue. Recently, we reported the thermosensitive catalytic activity of the iron porphyrin bound nanosphere in aqueous solution. ${ }^{5}$ In this paper, we focused on the recyclability and solvent effect of cyclohexene oxidation by iron porphyrin bound core-shell nanosphere.

\section{Experimental Section}

Materials. $N$-isopropylacrylamide (NIPAAm) (Acros) was recrystallized from hexane. Styrene, methylene bisacrylamide (MBA), vinyl pyridine, 2,2'-azobis(2-methylpropionamidine) dihydrochloride (V50), acetonitrile, dichloroethane and n-decane were used as received from Aldrich Chemical Co. Cyclohexene (Aldrich) was purified by filtration through silica gel prior to use. Sodium salt of 5,10,15,20-tetrakis(2,6difluoro-3-sulfonatophenyl)porphinato iron(III) [Fe(TDFPPS) $]^{3+}$ and sodium salt of 5,10,15,20-tetrakis(2,6-dichloro-3sulfonatophenyl)porphinato iron(III) $[\mathrm{Fe}(\mathrm{TDCPPS})]^{3+}$ were used as received from Mid-Century Chemicals. Iodosylbenzene $(\mathrm{PhIO})$ was prepared from iodobenzene diacetate by a literature method. ${ }^{6}$

Instrumentation. Scanning electron microscopy (SEM) (JEOL JSM-5200), dynamic light scattering (DLS) (ALV5000/60X0) and UV-vis spectrophotometer (Hewlett Packard 8453) were used to characterize the nanosphere. Products for cyclohexene oxidation were analyzed by a gas chromatography (Agilent Technologies $6890 \mathrm{~N}$ ) equipped with a flame ionization detector (FID) using a 25-m capillary column.

Preparation of the metalloporphyrin bound core-shell nanosphere. The preparation of core-shell nanospheres was reported elsewhere. ${ }^{7,8}$ The core was prepared by copolymerizing styrene $\left(18.0 \mathrm{~g}, 1.7 \times 10^{-1} \mathrm{~mol}\right)$ and $\mathrm{N}$-isopropyl acryl amide (NIPAAm) $\left(2.0 \mathrm{~g}, 1.8 \times 10^{-2} \mathrm{~mol}\right)$ in water at $70{ }^{\circ} \mathrm{C}$ using 2,2'-azobis(2-methylpropionamidine) dihydrochloride (V50) $\left(0.2 \mathrm{~g}, 7.2 \times 10^{-1} \mathrm{mmol}\right)$ as an initiator. The core particles were separated by ultrafiltration with a cellulose membrane (cut-off molecular weight: 100,000), and then freeze-dried. On the core particle $(3.0 \mathrm{~g})$, NIPAAm $(1.83 \mathrm{~g}$, $1.6 \times 10 \mathrm{mmol})$, vinyl pyridine $\left(0.032 \mathrm{~g}, 2.9 \times 10^{-1} \mathrm{mmol}\right)$ and methylene bisacrylamide (MBA) $\left(0.09 \mathrm{~g}, 5.8 \times 10^{-1}\right.$ mmol) were copolymerized in water at $70{ }^{\circ} \mathrm{C}$ using V50 $\left(0.0336 \mathrm{~g}, 1.2 \times 10^{-1} \mathrm{mmol}\right)$ as an initiator. The core-shell 
nanospheres were separated by ultrafiltration and freezedried. To prepare the metalloporphyrin bound core-shell nanosphere, the core-shell nanospheres $(0.5 \mathrm{~g})$ was added to the iron porphyrin $(0.10 \mathrm{mM})$ aqueous solution $(100 \mathrm{~mL})$. The metalloporphyrin bound core-shell nanosphere was washed three times with water $(100 \mathrm{~mL})$ followed by ultrafiltration. The filtered nanosphere was then freezedried.

Loading of iron porphyrins to the core-shell nanosphere. The loading of iron porphyrins to the core-shell nanosphere was determined by monitoring the disappearance of Soret bands at $410 \mathrm{~nm}$ for $[\mathrm{Fe}(\mathrm{TDCPPS})]^{3+}$ or 385 $\mathrm{nm}$ for $[\mathrm{Fe}(\mathrm{TDFPPS})]^{3+}$. To the iron porphyrin aqueous solution $(0.1 \mathrm{mM}, 100 \mathrm{~mL})$ was core-shell nanospheres $(0.5$ g) added. Then, the reaction mixture was stirred at room temperature for 24 hours. The reaction mixture was centrifuged at 13,000 rpm for $15 \mathrm{~min}$ and the supernatant was checked with UV-vis spectrophotometer.

Binding isotherm of iron porphyrins to the core-shell nanosphere. The iron porphyrin [Fe(TDCPPS)] aqueous solution was prepared in a concentration range of 0.01-0.2 $\mathrm{mM}$. Core-shell nanospheres $(75 \mathrm{mg})$ were suspended in the iron porphyrin [Fe(TDCPPS)] aqueous solution $(1.5 \mathrm{~mL})$ and the mixture was stirred for 24 hours at room temperature. The reaction mixture was centrifuged at 13,000 rpm for $15 \mathrm{~min}$ and the supernatant was checked with UV-vis spectrophotometer.

Catalytic oxidation of cyclohexene. In a typical oxidation reaction, iodosylbenzene $\left(\mathrm{PhIO}, 2.5 \times 10^{-2} \mathrm{mmol}\right)$ and cyclohexene $\left(2.5 \times 10^{-2} \mathrm{mmol}\right)$ were added to a reaction solution containing an iron porphyrin catalyst $\left(2.5 \times 10^{-4}\right.$ $\mathrm{mmol})$ or an iron porphyrin bound nanosphere $\left(2.5 \times 10^{-4}\right.$ $\mathrm{mmol})$ in acetonitrile/water cosolvent $(2.0 \mathrm{~mL})$. The reaction mixture was stirred for 7 hours at room temperature. The products were extracted with dichloroethane $(2.0 \mathrm{~mL})$ and analyzed by gas chromatography using decane as an internal standard. $^{9}$

Recycling experiment performed with the nanocatalyst. The catalytic activities of Fe(TDCPPS) bound nanosphere and $\mathrm{Fe}$ (TDFPPS) bound nanosphere were compared in a solvent mixture $(2.0 \mathrm{~mL})$ of acetonitrile and water $(v / v=$ $1: 1)$. The catalyst that showed higher yield between Fe(TDCPPS) bound nanosphere or Fe(TDFPPS) bound nanosphere was chosen and then recycling experiments were performed. Iodosylbenzene $\left(2.5 \times 10^{-2} \mathrm{mmol}\right)$ and cyclohexene $\left(2.5 \times 10^{-2} \mathrm{mmol}\right)$ were added to a solution containing iron porphyrin bound nanosphere $\left(2.5 \times 10^{-4} \mathrm{mmol}\right)$ in a solvent mixture $(2.0 \mathrm{~mL})$ of acetonitrile and water $(v / v=1$ : 1). The reaction mixture was stirred for 7 hours at room temperature. An aliquot $(0.2 \mathrm{~mL})$ of the reaction mixture was then extracted with dichloroethane $(0.2 \mathrm{~mL})$ and analyzed by gas chromatography using decane as an internal standard. ${ }^{9}$ The remaining reaction mixture was centrifuged at $13,000 \mathrm{rpm}$ for $30 \mathrm{~min}$ to separate the nanocatalyst and the supernatant was decanted. The nanocatalyst was rinsed with acetonitrile/water $(v / v=1: 1)$ three times and then vacuum dried overnight. The oxidation reaction was repeated three times by adding cyclohexene and $\mathrm{PhIO}$ under the identical conditions, and the product yields were analyzed by the same procedure described above.

\section{Results and Discussion}

Synthesis and characterization of iron porphyrin bound core-shell nanospheres. Scheme 1 shows the synthetic scheme of the metalloporphyrin bound core-shell nanosphere.

The preparation of iron porphyrin anchored core-shell nanospheres was followed by FT-IR (Figure 1). The relative increase in the amide I $\left(1650 \mathrm{~cm}^{-1}\right)$ and amide II (1550 $\mathrm{cm}^{-1}$ ) bands of the core-shell nanosphere to core showed the formation of poly(N-isopropyl acryl amide) shell. In the FTIR spectra, there was no difference between core-shell nanosphere and iron porphyrin anchored core-shell nanosphere. However, loading of iron porphyrins was confirmed by UVvis as would be discussed later.

The scanning electron microscopic images of core and core-shell nanospheres are shown in Figure 2. The diameter

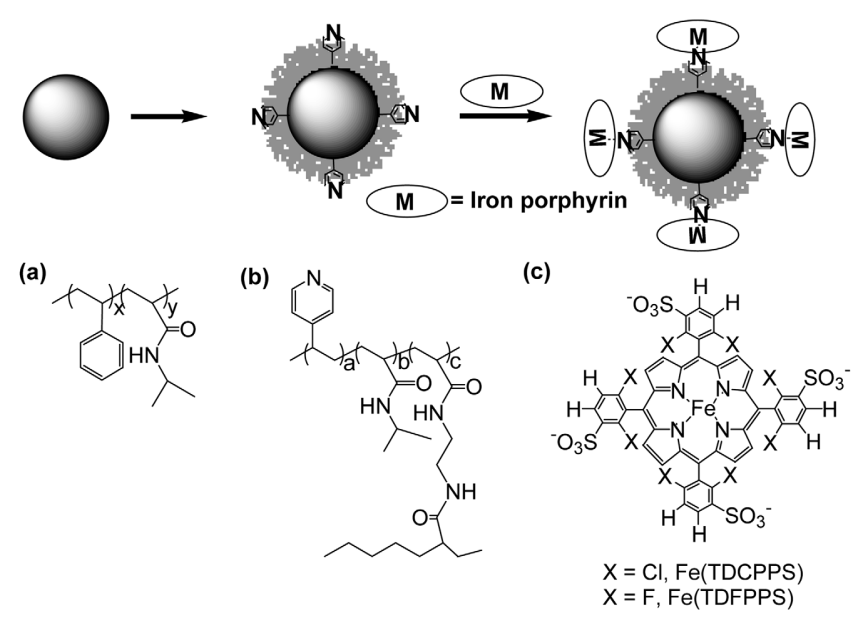

Scheme 1. Synthetic scheme of an iron porphyrin bound core-shell nanosphere, and structures of (a) core, (b) shell, and (c) iron porphyrins.

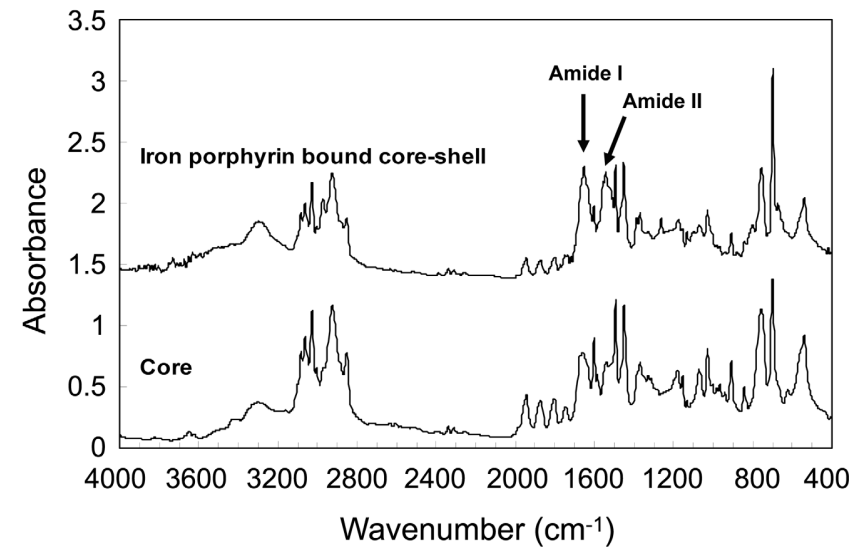

Figure 1. FT-IR spectra of the core and the iron porphyrin bound core-shell nanosphere. 
(a)

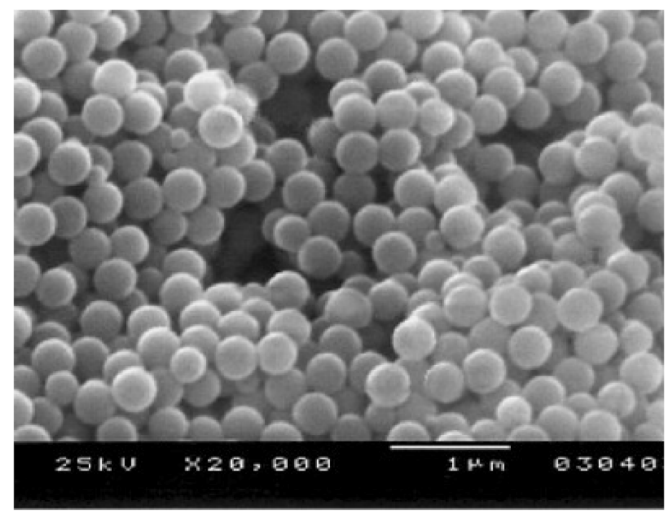

(b)

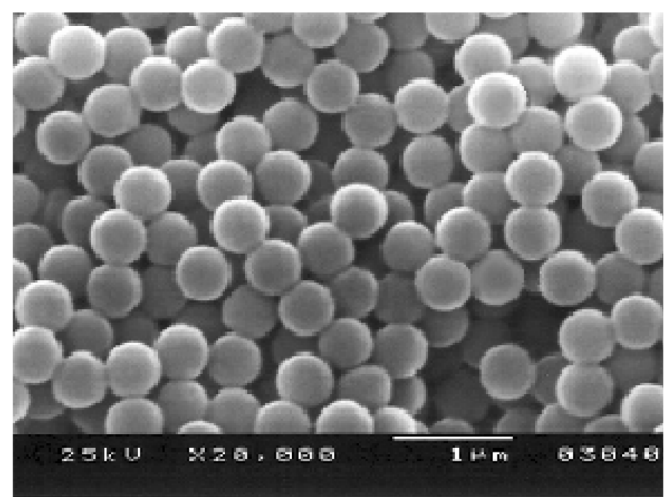

Figure 2. Scanning electron microscopic images of (a) core and (b) iron porphyrin bound core-shell nanospheres.

was $370 \mathrm{~nm}$ and $470 \mathrm{~nm}$, respectively. Instead of a nucleophilic substitution reaction to make traditional polymersupport catalyst of polystyrene beads, simple mixing of the aqueous solutions of catalysts and core-shell nanospheres can make the stable heterogeneous catalyst. The pyridinyl groups in the shell of the nanosphere bind to the iron porphyrin through coordination bonds.

The change in UV-vis spectra was investigated after adding the core-shell nanosphere to the iron porphyrin aqueous solution (Figure 3). The absorption band of iron porphyrin aqueous solutions at $385 \mathrm{~nm}$ was decreased due to the binding of the iron porphyrins to the nanosphere. UV-vis spectra also showed that the iron porphyrin was not loaded on the core-shell nanosphere without pyridinyl group, indicating the iron porphyrins are specifically anchored to pyridine moieties in the shell. The number of bound iron porphyrins on the core-shell nanosphere $(1.0 \mathrm{~g})$ was calculated to be $0.014 \mathrm{mmol}$ for Fe(TDCPPS) and 0.013 mmol for Fe(TDFPPS), respectively.

The binding isotherm of metalloporphyrin [Fe(TDCPPS)] on the nanosphere is shown in Figure 4. The binding site was saturated with iron porphyrins (substrate) when the concentration was larger than $0.08 \mathrm{mM}$. Assuming that 1) the monomer composition of the surface is the same as the feed composition of the monomer, and 2) the density of nanosphere is about $1.0 \mathrm{~g} / \mathrm{cm}^{3}$, the average number of pyridines on a nanosphere surface is calculated to be $4.6 \times$ $10^{5}$. Based on the binding isotherm, the maximum number (a)

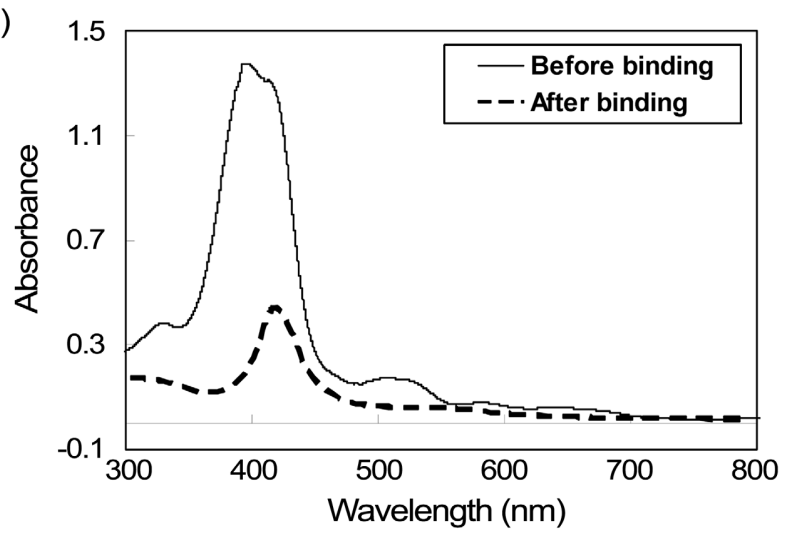

(b)

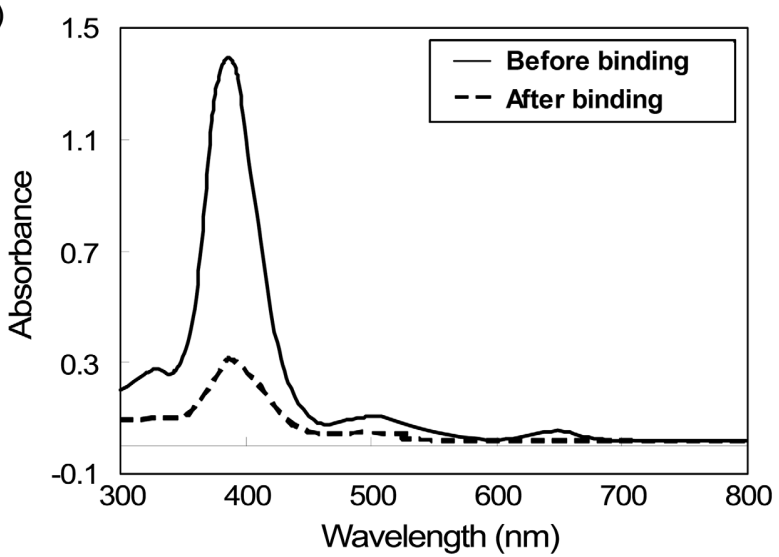

Figure 3. UV-vis spectral changes of (a) $[\mathrm{Fe}(\mathrm{TDCPPS})]^{3+}$ and (b) $[\mathrm{Fe}(\mathrm{TDFPPS})]^{3+}$ before and after the addition of core-shell nanosphere to the iron porphyrin aqueous solutions.

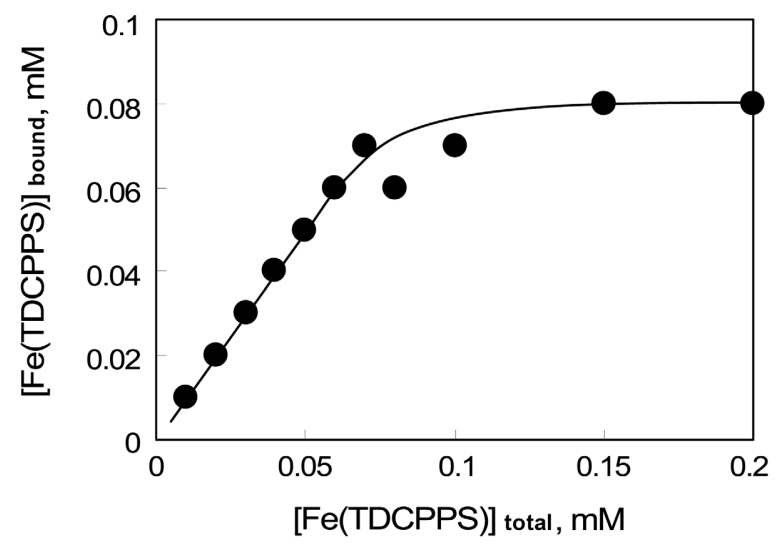

Figure 4. Binding isotherm of porphyrin catalyst [Fe(TDCPPS)] on the core-shell nanosphere.

of iron porphyrin on a nanosphere is calculated to be $3.5 \times$ $10^{5}$. The binding curve was analyzed by a Scatchard plot. ${ }^{10}$

$$
[\mathrm{S}]_{\text {bound }} /[\mathrm{S}]_{\text {free }}=\mathrm{c} / \mathrm{K}_{\mathrm{D}}-[\mathrm{S}]_{\text {bound }} / \mathrm{K}_{\mathrm{D}}
$$

$[\mathrm{S}]_{\text {bound }}$ and $[\mathrm{S}]_{\text {free }}$ are bound metalloporphyrin concentration and unbound (free) substrate concentration, respectively. $\mathrm{c}$ is a constant related to the apparent maximum number of binding sites and $\mathrm{K}_{\mathrm{D}}$ is the dissociation constant between substrate and binding sites. $K_{D}$ is a measure of binding 
strength between substrates and binding sites. For example, $\mathrm{K}_{\mathrm{D}}$ value of Con-A to glucose is $1.5 \times 10^{-3} \mathrm{~mol} / \mathrm{L} .{ }^{10}$ From the slope of the Scatchard plot, the $\mathrm{K}_{\mathrm{D}}$ was calculated to be $9.3 \times 10^{-3} \mathrm{~mol} / \mathrm{L}$. The linear Scatchard plot $\left(\mathrm{r}^{2}=0.94\right)$ indicates the binding sites can be seen as a class. ${ }^{11}$

Catalytic oxidation of cyclohexene. The oxidation of cyclohexene by homogeneous iron porphyrin catalyst [Fe(TDCPPS)] yielded $13-39 \%$ cyclohexene oxide, trace amount (less than 3\%) of 2-cyclohexen-1-one, and 2cyclohexen-1-ol in a solvent composition range of 25-75 vol. \% (acetonitrile/water). The yield was maximal at 50/50 solvent composition of acetonitrile and water. This fact seems to be caused by the solubility of reactant (cyclohexene) and the catalyst (iron porphyrin) that was optimal to increase the product yield at the intermediate solvent composition. The cyclohexene oxidation by iron porphyrin bound nanosphere showed similar trends when the acetonitrile fraction is in this solvent composition range, indicating that the intrinsic reactivity of metalloporphyrin catalyst is not significantly changed after the coordination bonded to the core-shell nanosphere.

Based on the above results, 5,10,15,20-tetrakis(2,6difluoro-3-sulfonatophenyl) porphinato) iron(III) [Fe(TDFPPS)] bound nanosphere was also studied at 50/50 $(\mathrm{v} / \mathrm{v})$ acetonitrile/water cosolvent. Interestingly, it showed $81 \%$ yield of cyclohexene oxide, and a trace amount of 2cyclohexen-1-one and 2-cyclohexen-1-ol. To check the recyclability, the iron porphyrin bound nanosphere was recovered by ultracentrifugation. The recycled iron porphyrin bound nanosphere was used as a catalyst. Cyclohexene oxide was formed with a yield of $61 \%$ at the second cycle, and $58 \%$ for third cycle, indicating the recyclability of the catalyst with a little loss of catalytic activity.

Polystyrene supported ruthenium porphyrin catalyst was reported for cyclohexene oxidation. The nanocatalyst was recycled four times by ultracentrifugation without significant loss of catalytic activity. However, it produces mixture of products such as $66 \%$ cyclohexene oxide, $25 \% 2-$ cyclohexen-1-one, and 7.5\% 2-cyclohexen-1-ol. ${ }^{13}$ In case of 2,6-Cl-TPP (TPP = tetraarylporphyrin) attached to nanoporous silica lost half of its original activity after being used two times. ${ }^{14}$ Compared with those heterogeneous systems, our porphyrin bound core-shell nanosphere showed improved stereoselective reactivity as well as recyclability.

\section{Conclusions}

The facile preparation method of heterogeneous porphyrin catalyst was developed by simple mixing the iron porphyrin with core-shell nanosphere. Iron porphyrin bound to the core-shell nanosphere showed similar activity to the homogeneous iron porphyrin in a solvent composition range of $25-75 \%$ acetonitrile/water (v/v). The catalyst showed recyclable and stereoselective catalytic activity for the oxidation of cyclohexene. The introduction of the ligand on the surface of the core-shell nanosphere, followed by coordination bonding to the catalyst, can be a facile method to prepare the polymer supported catalyst.

Acknowledgements. This work was supported by the Korea Science and Engineering Foundation (Grant No: R012002-000-00274-0) and Ministry of Science and Technology of Korea through Creative Research Initiative Program.

\section{References}

1. Merrifield, R. B. Angew. Chem. 1985, 97, 801.

2. Bergbreiter, D. E. J. Polym. Sci. Part A: Polym. Chem. 2001, 39, 2351.

3. Fujita, M.; Costas, M.; Que, L. Jr. J. Am. Chem. Soc. 2003, 125, 9912.

4. de Visser, S. P.; Kaneti, J.; Neumann, R.; Shaik, S. J. Org. Chem. 2003, 68, 2903.

5. Choi, B. G.; Song, R.; Nam, W.; Jeong, B. Chem. Commun. 2005, 23, 2960.

6. Saltzman, H.; Sharefkin, J. G. Organic Syntheses; Wiley: New York, 1973; Collect. Vol. V, p 658.

7. Makino, K.; Yamamoto, S.; Fujimoto, K.; Kawaguchi, H.; Ohshima, H. J. Colloid Interface Sci. 1994, 166, 251.

8. Duracher, D.; Sauzedde, F.; Elaissari, A.; Perrin, A.; Pichot, C. Colloid Polym. Sci. 1998, 276, 219.

9. Nam, W.; Oh, S.-Y.; Sun, Y. J.; Kim, J.; Kim, W. K.; Woo, S. K.; Shin, W. J. Org. Chem. 2003, 68, 7903.

10. Seong, H.; Lee, H.; Park, K. J. Biomater. Sci. Polymer Ed. 2002, $13,637$.

11. Martin, A.; Swarbrick, J.; Cammarata, A. Physical Pharmacy, $3^{\text {rd }}$ ed.; Lea \& Febiger: Philadelphia, 1983; p 335.

12. Wang, G.; Kuroda, K.; Enoki, T.; Grosberg, A.; Masamune, S.; Oya, T.; Takeoka, Y.; Tanaka, T. Proc. Nat'l. Acad. Sci. USA 2000, 97, 9861.

13. Yu, X.-Q.; Huang, J.-S.; Yu, W.-Y.; Che, C.-M. J. Am. Chem. Soc. 2000, 122, 5337.

14. Liu, C.-J.; Yu, W.-Y.; Li, S.-G.; Che, C.-M. J. Org. Chem. 1998, 63, 7364 . 